\title{
Association Between Functional PSMD10 Rs111638916 Variant Regulated by MiR-505 and Gastric Cancer Risk in a Chinese Population
}

\author{
Yong Liu ${ }^{a}$ Jianzhong Xua Min Jiang ${ }^{a}$ Lingna Nia Yun Chen ${ }^{b}$ Yang Ling \\ aDepartment of Oncology, Changzhou Cancer Hospital of Soochow University, Changzhou, Jiangsu \\ Province, ${ }^{b}$ Department of Immunology, Nanjing Medical University, Nanjing, Jiangsu Province, P.R.China
}

\author{
Key Words \\ Genotype $\cdot$ MiR-505 • 3'-UTR • Tumor growth • Metastasis
}

\begin{abstract}
Background/Aims: Gankyrin is an oncoprotein involved in regulating the cell cycle through protein-protein interactions with cyclin-dependent kinase 4 and p53. However, its association with gastric cancer (GC) risk has not yet been determined. In this study, we investigated micro RNA (miRNA)-associated single nucleotide polymorphisms (SNPs) in the 3'-untranslated region (UTR) of the gankyrin gene PSMD10 to clarify the relationship between these SNPs and miRNAs in Chinese patients with GC. Methods: We performed a case-control study including 857 GC patients and 748 cancer-free controls. PSMD10 expression was investigated using genotyping, real-time polymerase chain reaction, cell transfection, and dual luciferase reporter assays. Results: Patients with histories of smoking, alcohol consumption, and cancer were more susceptible to GC than controls. The SNP rs111638916 in the PSMD10 3'-UTR was identified as a risk factor for GC and acted as a tumor promoting factor. SNP rs 111638916 was also regulated by miR-505, resulting in up-regulation of gankyrin expression in patients with GA and AA genotypes. Carriers of the GA and AA genotypes also presented with larger tumors and had a higher risk of metastasis. Conclusion: The PSMD10 rs111638916 SNP is highly associated with an increased risk of GC in Chinese patients, and could serve as a novel biomarker for this disease.
\end{abstract}

Copyright $@ 2015$ S. Karger AG, Basel

\section{Introduction}

Gastric cancer (GC) is one of the most common malignancies and an important cause of mortality worldwide, especially in Asia (e.g., China, Japan, and South Korea)[1], with about $42 \%$ of all cases occurring in China. The prognosis of GC is currently determined primarily

Dr. Yang Ling and Dr. Yun Chen

KARGER 125
Department of Oncology, Changzhou Cancer Hospital of Soochow University, Changzhou, Jiangsu Province, (P.R. China); and Department of Immunology, Nanjing Medical University, 140 Hanzhong Road, Nanjing, Jiangsu Province, (P.R. China) Tel. +862586862901, E-Mail chenyun@njmu.edu.cn 
on the bases of clinical data and the pathologic stage of the patient at the time of diagnosis and treatment $[2,3]$. Recent surgical, chemotherapeutic, immunologic, and radiation approaches have resulted in significant improvements in survival of patients with localized disease [4]; However, the successful management of patients with GC is still hampered by a lack of highly sensitive and specific biomarkers capable of predicting prognosis and the likelihood of metastasis [5]. Etiological studies have suggested that environmental factors, including diets high in salted and nitrated foods, tobacco use, alcohol consumption, and Helicobacter pylori infection are all highly associated with GC [6-8]. However, only some people exposed to the same environmental risk factors eventually develop GC, suggesting that genetic factors may also be involved in the etiology of this disease.

MicroRNAs (miRNAs) are small, non-coding RNA molecules 19-25 nucleotides long that regulate gene expression by transcriptional repression of mRNA functions [9]. MiRNAs can negatively regulate expression of their target genes by hybridizing to incomplete complementary sequences in the 3'-untranslated region (UTR) of the mRNA, resulting in degradation of the target mRNA or repression of mRNA translation $[10,11]$. Their potential effects on miRNA expression and the subsequent impact on mRNA transcription mean that miRNA single nucleotide polymorphisms (SNPs) or miRNA-related SNPs, such as SNPs in the miRNA-binding sites in the 3'-UTR of their target genes, have been implicated as crucial genetic factors in susceptibility to various cancers[12-14]. Epigenetic alterations, including aberrant DNA methylation changes, may also play an important role in gastric carcinogenesis, as indicated by the increased hyper methylation of tumor suppressor genes in patients with GC [5]. Given their important functions in cancer initiation and progression, methylation changes are currently being investigated as potential biomarkers for early cancer detection, and prediction of cancer progression and chemotherapeutic sensitivity. A recent study found that significant methylation changes in PSMD10, which encodes gankyrin, were more frequent in gastric carcinoma and GC with metastasis compared with normal samples, suggesting that PSMD10 may act as a tumor suppressor gene, as well as an oncogene, as previously reported $[15,16]$. Gankyrin was initially purified and characterized by Tanaka and coworkers as the p28 component of the regulatory subunit of the $26 \mathrm{~S}$ proteasome, which is an ATP-dependent protease responsible for the degradation of proteins [17]. Ectopically expressed gankyrin was shown to bind retinoblastoma protein (Rb), but not the pRb-related proteins p107 and p130 in vitro and in vivo, providing an initial glimpse into the role of gankyrin in tumorigenesis [18]. Overexpression of gankyrin also conferred tumorigenicity to NIH/3T3 cells and inhibited apoptosis in cultured human tumor cells exposed to chemotherapeutic agents [19].

We therefore considered that the role of gankyrin in tumorigenesis might be affected by genetic factors. No SNPs have yet been identified in PSMD10. We therefore investigated SNPs in the 3'-UTR using bioinformatics software (http://www.bioguo.org/miRNASNP/). We detected candidate SNPs that could affect PSMD10 gene regulation via miRNAs. We identified rs111638916 as the only SNP potentially regulated by miR-505 and further investigated its allele distribution in a case-control study.

\section{Materials and Methods}

\section{Study subjects}

A total of 857 GC cases from Changzhou Cancer Hospital of Soochow University (Changzhou, Jiangsu Province, China) and sex- and age-matched 748 controls were included in this study. Patients were consecutively recruited between February 2010 and January 2014 at Changzhou Cancer Hospital. All cases are incident ones during enrollment of the current case-control study. The diagnosis of all patients was histological confirmed. A face-to-face questionnaire was administered to collect demographic data and environmental exposure information, including alcohol use and cigarette consumption status as well as family cancer history. Subjects who smoked one cigarette per day for more than one year were considered as smokers. Individuals were considered as alcohol drinkers, if they drank at least once every week. 60 
pairs of GC tissues and adjacent normal tissues to the tumors were obtained from surgically removed specimens of patients. The normal tissues sampled at least $2 \mathrm{~cm}$ away from the margin of the tumor. At recruitment, the informed consent was obtained from each subject. All participants have provided their written informed consents to participate in this study. This study was approved by the institutional Review Board of Changzhou Cancer Hospital.

\section{Genotype}

The rs111638916 G>A polymorphism was genotyped through the PCR-restriction fragment length polymorphism (RFLP) method as described previously. The PCR reactions were carried out in a total volume of $5 \mu \mathrm{L}$ containing TaqMan Universal Master Mix, 80X SNP Genotyping AssayMix, Dnase-free water and 10ng genomic DNA. The PCR conditions were $2 \mathrm{~min}$ at $50^{\circ} \mathrm{C}, 10 \mathrm{~min}$ at $95^{\circ} \mathrm{C}$, followed by 40 cycles at $95^{\circ} \mathrm{C}$ for $15 \mathrm{sec}$ and $60^{\circ} \mathrm{C}$ for $1 \mathrm{~min}$. The 384-well ABI 7900HT Real Time PCR System. A $10 \%$ random sample was reciprocally examined by different persons, and the reproducibility was $100 \%$.

Real time PCR assay

Real time polymerase chain reaction (RT-PCR) was performed to determine whether the G to A mutation changed the expression level of Gankyrin. The amplification conditions were $95^{\circ} \mathrm{C}$ for 10 minutes, followed by 40 cycles of $95^{\circ} \mathrm{C}$ for 30 seconds, $55^{\circ} \mathrm{C}$ for 40 seconds, and $72^{\circ} \mathrm{C}$ for 30 seconds, and finally $4^{\circ} \mathrm{C}$ for 30 minutes for cooling as described [14].

\section{Cell lines and cell culture}

GC cell lines SGC-7901 and MKN-45 were purchased from the Chinese Academy of Sciences Cell Bank. All cells were cultured in Dulbecco's Minimum Essential Medium (DMEM) purchased from Gibco (CA, USA) supplemented with $10 \%$ fetal bovine serum (Invitrogen, Carlsbad, USA) and grown in humidified $5 \%$ $\mathrm{CO} 2$ at $37^{\circ} \mathrm{C}$. MiR-505 mimics and normal control were obtained from Genepharma (Shanghai, China).The transfection was conducted by using Lipofectamine 2000 (Invitrogen Corp, CA, USA).

\section{Prediction of miRNAs binding to the SNP}

We conducted the bioinformatics analysis (http://www.bioguo.org/miRNASNP/) to predict the related SNPs in the 3'UTR of Gankyrin. For the SNPs in miRNA seed regions, two different methods were used to predict the target sites for the wild-type miRNAs and SNP-miRNAs. These resulted in four groups of target gene data, which are recorded as WT (target genes of wild-type miRNAs processed by TargetScan), WM (target genes of wild-type miRNAs processed by miRanda), ST (target genes of SNP-miRNAs processed by TargetScan), and SM (target genes of SNP-miRNAs processed by miRanda). If one miRNA/target pair exists in both WT and WM, but not in either ST or SM, we called this miRNA/target pair loss. On the contrary, if one miRNA/target pair was predicted in both ST and SM, but neither in WT nor WM, we defined the SNP-miRNA gained the target gene. In addition, for each miRNA/target loss or gain pair, we obtained the sequence $( \pm 50 \mathrm{bp})$ of target site and used RNAhybrid to calculate the minimum hybridization energy of the miRNA-target interaction. Generally, more energy change would affect the miRNA-target interaction more sharply. The binding energy changes between wild-type miRNA/target and SNP-miRNA/target were provided in our database as additional information for users making further judgments.

\section{Construction of luciferase-based reporter plasmids}

A full length fragment of the 3'UTR containing either G or A allele of rs111638916 was amplified. The PCR product was cloned into the pGL3-promoter luciferase-based plasmid (Promega, CA, USA). The amplified fragment was verified by DNA sequencing.

\section{Dual-luciferase reporter assay}

For luciferase activity analysis, SGC-7901 and MKN-45 cells were co-transfected with 100ng of luciferase reporter constructs $5 \mathrm{ng}$ of the $\beta$-gal control plasmid and $10 \mathrm{pmol}$ of miRNAs with $1 \mu$ lipofectamine 2000 according to the manufacturer's instructions (Invitrogen, NY, USA). After incubation for 48h, we carried out the luciferase assay using the luciferase reporter assay system (Promega, Madison, WI) according to the manufacturer's protocol. Measurements of luminescence and absorbance of $\beta$-gal were performed on a luminometer (Glomax 20/20; Promega). Three independent experiments were performed in triplicate. 


\section{Statistical analysis}

Differences between cases and controls were evaluated by the Student's t-test for continuous variables and the $\chi 2$ test for categorical variables. The association between SNPs and GC risk was estimated by the OR and 95\% CI using the general genetic model. The potential gene-environment interaction was evaluated by logistic regression analysis and tested by comparing changes in deviance between the models of main effects with or without the interaction term. Comparisons between groups were analyzed by the $t$ test (twosided). All statistical analyses were performed using Statistical Package for Social Sciences software.

\section{Results}

The differences in the distribution of the selected variables among GC cases and controls are shown in Table 1 . There were no significant differences in age $(\mathrm{P}=0.628)$, sex $(\mathrm{P}=$ $0.758)$, and parental smoking status ( $\mathrm{P}=0.537)$ between the case and control groups, but alcohol consumption was more common among cases than controls $(\mathrm{P}=0.029)$. As expected, a family history of cancer was also more common among patients than controls $(\mathrm{P}<0.0001)$. However, all the above variables were further adjusted for any residual confounding effect in the subsequent multivariate logistic regression analysis.

The genotype distributions of PSMD10 rs111638916 G>A in cases and controls are presented in Table 2. The genotype distribution in the controls was in agreement with the Hardy-Weinberg equilibrium, and the distributions differed significantly between the case and control groups $(\mathrm{P}<0.0001)$. Univariate logistic regression analysis showed an OR of 2.39 and a 95\%CI of 1.25-1.82 for frequency of the GA genotype between the two groups, and an OR of 1.32 and a $95 \%$ CI of 1.15-2.44, respectively, for the AA genotype. The result was more significant in the dominant model $(\mathrm{OR}=$ $2.10,95 \% \mathrm{CI}=1.27-1.79, \mathrm{P}<$ 0.0001 ), given that the combined genotype CT/TT was associated with an increased risk of GC.

We also observed an association between the rs111638916 polymorphism

Table 1. Frequency distributions of selected variables in patients and cancer-free controls. *Two-sided chi-square test for either genotype distributions or allele frequencies between cases and controls

\begin{tabular}{|c|c|c|c|c|c|}
\hline \multirow{2}{*}{ Variables } & \multicolumn{2}{|c|}{ Cases $(n=857)$} & \multicolumn{2}{|c|}{ Controls $(n=748)$} & \multirow{2}{*}{$P$} \\
\hline & $N$ & $\%$ & $N$ & $\%$ & \\
\hline \multicolumn{6}{|l|}{ Age (years) } \\
\hline$\leq 60$ & 446 & 52.04 & 401 & 53.61 & 0.628 \\
\hline$>60$ & 411 & 47.96 & 347 & 46.39 & \\
\hline \multicolumn{6}{|l|}{ Gender } \\
\hline Male & 426 & 49.71 & 386 & 51.60 & 0.758 \\
\hline Female & 431 & 50.29 & 362 & 48.40 & \\
\hline \multicolumn{6}{|c|}{ Parental smoking status } \\
\hline Negative & 421 & 49.12 & 379 & 50.67 & 0.537 \\
\hline Positive & 436 & 50.88 & 369 & 49.33 & \\
\hline \multicolumn{6}{|c|}{ Parental drinking status } \\
\hline Negative & 407 & 47.49 & 396 & 52.94 & 0.029 \\
\hline Positive & 450 & 52.51 & 352 & 47.06 & \\
\hline \multicolumn{6}{|c|}{ Family history of cancer } \\
\hline Negative & 368 & 42.94 & 427 & 57.09 & $<0.0001$ \\
\hline Positive & 489 & 57.06 & 321 & 42.91 & \\
\hline \multicolumn{6}{|c|}{ Differentiation grade } \\
\hline Well & 268 & 31.27 & & & \\
\hline Moderate & 318 & 37.11 & & & \\
\hline Poorly & 271 & 31.62 & & & \\
\hline \multicolumn{6}{|c|}{ Tumor Size $(\mathrm{cm})$} \\
\hline$\leq 3 \mathrm{~cm}$ & 491 & 57.29 & & & \\
\hline$>3 \mathrm{~cm}$ & 366 & 42.71 & & & \\
\hline \multicolumn{6}{|c|}{ Tumor Number } \\
\hline Solitary & 382 & 44.57 & & & \\
\hline Multiple & 475 & 55.43 & & & \\
\hline \multicolumn{6}{|c|}{ Tumor Capsular } \\
\hline Incomplete & 407 & 47.49 & & & \\
\hline Complete & 450 & 52.51 & & & \\
\hline \multicolumn{6}{|l|}{ Metastasis } \\
\hline Yes & 397 & 46.32 & & & \\
\hline No & 460 & 53.68 & & & \\
\hline
\end{tabular}


Table 2. Genotype frequencies of the PSMD10 rs111638916 G>A polymorphism among GC cases

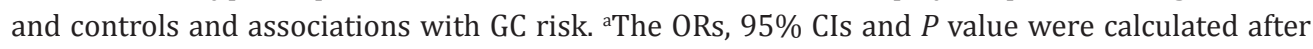
adjusting for age, gender, parental smoking, drinking and house painting status

\begin{tabular}{lcccccc}
\hline Genotype & \multicolumn{2}{c}{ Cases $(n=857)$} & \multicolumn{2}{c}{ Controls $(n=748)$} & Adjusted OR & Adjusted $P$ Value \\
& $n$ & $\%$ & $n$ & $\%$ & $(95 \% \mathrm{CI})^{\mathrm{a}}$ & A \\
\hline GG & 416 & 48.54 & 497 & 66.44 & 1.00 & $<0.0001$ \\
GA & 366 & 42.71 & 183 & 24.47 & $2.39(1.25-1.82)$ & \\
AA & 75 & 8.75 & 68 & 9.09 & $1.32(1.15-2.44)$ & \\
A carrier & 441 & 51.46 & 251 & 33.56 & $2.10(1.27-1.79)$ & $<0.0001$ \\
\hline
\end{tabular}

Table 3. Stratified analysis of rs111638916 genotype with clinicopathological parameters of GC. ${ }^{*}$ Two-sided chi-square test for either genotype distributions or allele frequencies between cases and controls

\begin{tabular}{|c|c|c|c|c|c|c|c|}
\hline \multirow{2}{*}{ Feather } & \multicolumn{4}{|c|}{ Genotype } & \multirow{2}{*}{$\begin{array}{l}\mathrm{GA} \text { vs GG } \\
P \text { Value }\end{array}$} & \multirow{2}{*}{$\begin{array}{l}\text { AA vs GG } \\
P \text { Value }\end{array}$} & \multirow{2}{*}{$\begin{array}{c}\text { A carrier vs GG } \\
P \text { Value }\end{array}$} \\
\hline & GG & GA & $\mathrm{AA}$ & A carrier & & & \\
\hline \multicolumn{8}{|c|}{ Age (years) } \\
\hline$\leq 60$ & 209 & 178 & 76 & 254 & 0.30 & 0.08 & 0.12 \\
\hline$>60$ & 157 & 157 & 80 & 237 & & & \\
\hline \multicolumn{8}{|l|}{ Gender } \\
\hline Male & 213 & 163 & 87 & 250 & 0.77 & 0.27 & 0.78 \\
\hline $\begin{array}{l}\text { Female } \\
\text { TMN gra }\end{array}$ & 185 & 148 & 61 & 209 & & & \\
\hline I-II & 264 & 103 & 51 & 154 & $<0.0001$ & $<0.0001$ & $<0.0001$ \\
\hline III-IV & 179 & 183 & 77 & 260 & & & \\
\hline \multicolumn{8}{|c|}{ Tumor Size $(\mathrm{cm})$} \\
\hline$\leq 3 \mathrm{~cm}$ & 217 & 104 & 73 & 177 & 0.004 & $<0.0001$ & $<0.0001$ \\
\hline \multicolumn{8}{|c|}{ Tumor location } \\
\hline $\mathrm{U}$ & 236 & 132 & 61 & 193 & 0.09 & 0.71 & 0.23 \\
\hline M & 218 & 158 & 52 & 210 & & & \\
\hline \multicolumn{8}{|c|}{ Metastasis } \\
\hline Yes & 158 & 168 & 137 & 305 & $<0.0001$ & $<0.0001$ & $<0.0001$ \\
\hline No & 243 & 104 & 47 & 151 & & & \\
\hline
\end{tabular}

and clinical features in GC patients (Table 3). The combined genotype GA/AA and the A allele were both associated with a significantly increased risk of TNF grade, larger tumor size, and tumor metastasis. However, there was no significant association with patient age, sex, or tumor location.

Using real-time PCR, we detected a significant difference in PSMD10 gene expression levels in patients carrying GG, GA, and AA genotypes (Fig. 1A). The expression levels in the GA and AA carriers were higher than in the GG carriers $(P<0.01)$.

We investigated the effect of the rs 111638916 polymorphism on the interaction between the predicted miRNA (miR-505) and the $3^{\prime}$-UTR conservative sites in gankyrin mRNA by transient transfection in vitro. We measured the relative activities using a dual luciferase reporter assay system. The detailed sequence is presented in Fig. 1B. Co-transfection of the luciferase vector with the PSMD10 3'-UTR containing the mutated allele A and hsa-miR-505 into significantly changed the luciferase expression level compared with a reporter plasmid containing the wild-type allele. We therefore concluded that the rs111638916 polymorphism 


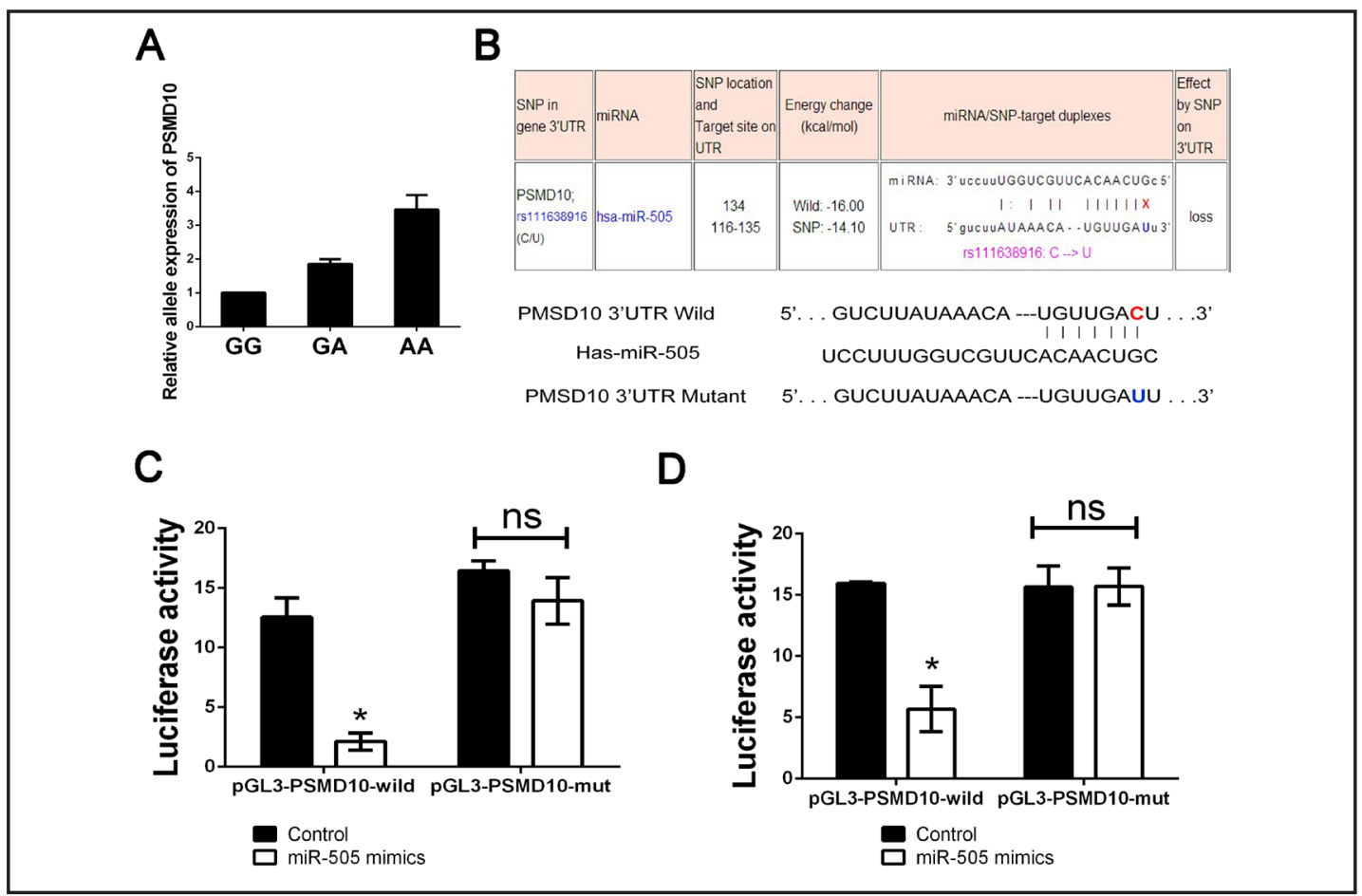

Fig. 1. MiR-505 binding with the GG genotype in the 3'UTR region reduced the expression of Gankyrin. (A) The expression level of Gankyrin was determined by RT-PCR in patients with GG, GA and AA genotypes. (B) Bioinformatics predicted the binding site between the miR-505 with Gankyrin. (C, D) Cells were co-transfected with miR-505 mimics or control for $48 \mathrm{~h}$ in SGC-7901 and MKN-45 cell lines. Data was presented as the mean \pm SEM. * indicates a significant difference $(\mathrm{P}<0.05)$, ns indicate no significance.

affected the binding affinity of miR-505 with the 3'-UTR in the PSMD10 mRNA, thus affecting post-transcriptional regulation and leading to abnormal expression of gankyrin.

\section{Discussion}

Gankyrin is an oncoprotein and a component of the 19S regulatory cap of the proteasome. It contains a series of $\alpha$-helices with a 33-amino acid ankyrin repeat [20,21]. Gankyrin is involved in the cell cycle through protein-protein interactions with the cyclin-dependent kinase CDK4 [22]. It also binds closely to the E3 ubiquitin ligase MDM2, which regulates the degradation of p53 and Rb protein, both of which are transcription factors involved in tumor suppression and which are mutated in many cancers.

Several reports have revealed that the gankyrin gene is overexpressed and acts as an oncogene in many tumors including liver cancer, colorectal cancer, breast cancer, and cholangiocarcinoma [21-24]. Most observations have been made in liver cancer and gankyrin overexpression is known to occur in most hepatocellular carcinomas. An increase in gankyrin expression in hepatocytes was one of the earliest molecular events in a chemical model of liver carcinogenesis $[20,25]$. However, knockdown of PSMD10 expression in hepatocellular carcinoma can result in reduced cell growth, enhanced $\mathrm{pRb}$ dephosphorylation, and caspase 8- and/or caspase 9-mediated apoptosis. In addition, disturbances in the balance between cell division and apoptosis by gankyrin overexpression during hepatic regeneration, causing inactivation of $\mathrm{pRb}$ and elimination of $\mathrm{p} 53$, could provide a mechanism for hepatocarcinogenesis. Gankyrin has also demonstrated a tumorigeneisis-promoting role in breast cancer. It was identified in normal breasts and was overexpressed in invasive breast cancers, and its overexpression was associated with extensive intraductal carcinoma and 
ErbB2 expression [26]. In addition, gankyrin deletion abrogated the increased metastatic potential of breast cancer cells under hypoxic conditions, partly through regulating E-cadherin [27]. Gankyrin was also found to maintain the stemness of colorectal cancer and control stem cell behavior by regulating the expression of stemness factors. Significant correlations were observed between gankyrin, vascular endothelial growth factor, and Nanog in colorectal adenomas [28].

MiRNAs not only negatively regulate expression of their target genes at the posttranscriptional level through binding to the 3'-UTRs of their target mRNAs [29-31], but are also affected by SNPs in the 3'-UTR region. SNPs in the 3 '-UTR region of a gene may affect the impact of miRNA on post-transcriptional regulation in relation to complex diseases. Increasing evidence indicates that SNPs located in miRNA-binding sites can decrease or increase target-mRNA translation through affecting miRNA binding to their target genes, and may be associated with susceptibility to cancers [14, 32]. In the present study, we predicted that the rs111638916 SNP in the $3^{\prime}$-UTR of the PSMD10 gene would affect the binding efficiency of miR-505. Furthermore, increased PSMD10 gene expression demonstrated a reduced suppressive effect of miR-505, providing support for increased gankyrin expression in human GC.

In conclusion, our findings have shown that the SNP rs111638916 in Gankyrin 3'-UTR, through disrupting the regulatory role of miR-505 in Gankyrin expression, rs111638916 in Gankyrin might act as a protective factor in the pathogenesis of GC.

\section{Acknowledgements}

This work was supported by grants from the National Natural Science Foundation (30901750 and 81272322 to Y.C.).

\section{Disclosure Statement}

The authors declare that they have no financial conflict of interest.

\section{References}

1 Chen L, Jiang M, Yuan W, Tang H: Prognostic value of mir-93 overexpression in resectable gastric adenocarcinomas. Acta Gastroenterol Belg 2012;75:22-27.

2 Arita T, Ichikawa D, Konishi H, Komatsu S, Shiozaki A, Shoda K, Kawaguchi T, Hirajima S, Nagata H, Kubota T, Fujiwara H, Okamoto K, Otsuji E: Circulating long non-coding rnas in plasma of patients with gastric cancer. Anticancer Res 2013;33:3185-3193.

3 Guo X, Jing C, Li L, Zhang L, Shi Y, Wang J, Liu J, Li C: Down-regulation of vezt gene expression in human gastric cancer involves promoter methylation and mir-43c. Biochem Biophys Res Commun 2011;404:622627.

4 McGee DJ, Mobley HL: Pathogenesis of helicobacter pylori infection. Curr Opin Gastroenterol 2000;16:2431.

5 Liu Z, Zhang J, Gao Y, Pei L, Zhou J, Gu L, Zhang L, Zhu B, Hattori N, Ji J, Yuasa Y, Kim W, Ushijima T, Shi H, Deng D: Large-scale characterization of DNA methylation changes in human gastric carcinomas with and without metastasis. Clin Cancer Res 2014;20:4598-4612.

6 Tsai KW, Wu CW, Hu LY, Li SC, Liao YL, Lai CH, Kao HW, Fang WL, Huang KH, Chan WC, Lin WC: Epigenetic regulation of mir-34b and mir-129 expression in gastric cancer. Int J Cancer 2011;129:2600-2610.

7 Lin LL, Huang HC, Juan HF: Revealing the molecular mechanism of gastric cancer marker annexin a4 in cancer cell proliferation using exon arrays. PLoS One 2012;7:e44615.

8 Hoepler W, Hammer K, Hammer J: Gastric phenotype in children with helicobacter pylori infection undergoing upper endoscopy. Scand J Gastroenterol 2011;46:293-298.

9 Zhu Y, Xia Y, Niu H, Chen Y: Mir-16 induced the suppression of cell apoptosis while promote proliferation in esophageal squamous cell carcinoma. Cell Physiol Biochem 2014;33:1340-1348. 
10 Zhang JX, Song W, Chen ZH, Wei JH, Liao YJ, Lei J, Hu M, Chen GZ, Liao B, Lu J, Zhao HW, Chen W, He YL, Wang HY, Xie D, Luo JH: Prognostic and predictive value of a microrna signature in stage ii colon cancer: A microrna expression analysis. Lancet Oncol 2013;14:1295-1306.

11 Ying SY, Chang DC, Lin SL: The microrna. Methods Mol Biol 2013;936:1-19.

12 Zhang J, Yu H, Zhang Y, Zhang X, Zheng G, Gao Y, Wang C, Zhou L: A functional tnfaip2 3'-utr rs8126 genetic polymorphism contributes to risk of esophageal squamous cell carcinoma. PLoS One 2014;9:e109318.

13 Wang K, Xu L, Pan L, Xu K, Li G: The functional brca1 rs799917 genetic polymorphism is associated with gastric cancer risk in a chinese han population. Tumour Biol 2015;36:393-397.

14 Wang Y, Zhou L, Chen J, Li J, He L, Wu P, Wang M, Tong N, Zhang Z, Fang Y: Association of the 3'utr foxo3a polymorphism rs4946936 with an increased risk of childhood acute lymphoblastic leukemia in a chinese population. Cell Physiol Biochem 2014;34:325-332.

15 Padmanabhan B, Adachi N, Kataoka K, Horikoshi M: Crystal structure of the homolog of the oncoprotein gankyrin, an interactor of rb and cdk4/6. J Biol Chem 2004;279:1546-1552.

16 Su B, Luo T, Zhu J, Fu J, Zhao X, Chen L, Zhang H, Ren Y, Yu L, Yang X, Wu M, Feng G, Li S, Chen Y, Wang H: Interleukin-1beta/iinterleukin-1 receptor-associated kinase 1 inflammatory signaling contributes to persistent gankyrin activation during hepatocarcinogenesis. Hepatology 2015;61:585-597.

17 Hwang JA, Yang HM, Hong DP, Joo SY, Choi YL, Park JH, Lazar AJ, Pollock RE, Lev D, Kim SJ: Gankyrin is a predictive and oncogenic factor in well-differentiated and dedifferentiated liposarcoma. Oncotarget 2014;5:9065-9078.

18 Zheng T, Hong X, Wang J, Pei T, Liang Y, Yin D, Song R, Song X, Lu Z, Qi S, Liu J, Sun B, Xie C, Pan S, Li Y, Luo X, Li S, Fang X, Bhatta N, Jiang H, Liu L: Gankyrin promotes tumor growth and metastasis through activation of il-6/stat3 signaling in human cholangiocarcinoma. Hepatology 2014;59:935-946.

19 Bai Z, Tai Y, Li W, Zhen C, Gu W, Jian Z, Wang Q, Lin JE, Zhao Q, Gong W, Liang B, Wang C, Zhou T: Gankyrin activates il-8 to promote hepatic metastasis of colorectal cancer. Cancer Res 2013;73:4548-4558.

20 Iakova P, Timchenko L, Timchenko NA: Intracellular signaling and hepatocellular carcinoma. Semin Cancer Biol 2011;21:28-34.

21 Lozano G, Zambetti GP: Gankyrin: An intriguing name for a novel regulator of p53 and rb. Cancer cell 2005;8:3-4.

22 Lim IK: Spectrum of molecular changes during hepatocarcinogenesis induced by den and other chemicals in fischer 344 male rats. Mech Ageing Dev 2002;123:1665-1680.

23 Nanaware PP, Ramteke MP, Somavarapu AK, Venkatraman P: Discovery of multiple interacting partners of gankyrin, a proteasomal chaperone and an oncoprotein--evidence for a common hot spot site at the interface and its functional relevance. Proteins 2014;82:1283-1300.

24 Kim KH, Lim HJ, Kim YJ, Kim SW, Kim YS, Tian C, Park K, Park TJ, Choung YH: The oncoprotein, gankyrin, is up-regulated in middle ear cholesteatoma. Acta Otolaryngol 2014;134:238-243.

25 Chen X, Zhang M, Liu LX: The overexpression of multidrug resistance-associated proteins and gankyrin contribute to arsenic trioxide resistance in liver and gastric cancer cells. Oncol Rep 2009;22:73-80.

26 Kim YH, Kim JH, Choi YW, Lim SK, Yim H, Kang SY, Chung YS, Lee GY, Park TJ: Gankyrin is frequently overexpressed in breast cancer and is associated with erbb2 expression. Exp Mol Pathol 2013;94:360-365.

27 Gao L, Xie H, Dong L, Zou J, Fu J, Gao X, Ou L, Xiang S, Song H: Gankyrin is essential for hypoxia enhanced metastatic potential in breast cancer cells. Mol Med Rep 2014;9:1032-1036.

28 Mine H, Sakurai T, Kashida H, Matsui S, Nishida N, Nagai T, Hagiwara S, Watanabe T, Kudo M: Association of gankyrin and stemness factor expression in human colorectal cancer. Dig Dis Sci 2013;58:2337-2344.

29 Valastyan S, Reinhardt F, Benaich N, Calogrias D, Szasz AM, Wang ZC, Brock JE, Richardson AL, Weinberg RA: A pleiotropically acting microrna, mir-31, inhibits breast cancer metastasis. Cell 2009;137:1032-1046.

30 Hyun S, Lee JH, Jin H, Nam J, Namkoong B, Lee G, Chung J, Kim VN: Conserved microrna mir-8/mir-200 and its target ush/fog2 control growth by regulating pi3k. Cell 2009;139:1096-1108.

31 Wang K, Diskin SJ, Zhang H, Attiyeh EF, Winter C, Hou C, Schnepp RW, Diamond M, Bosse K, Mayes PA, Glessner J, Kim C, Frackelton E, Garris M, Wang Q, Glaberson W, Chiavacci R, Nguyen L, Jagannathan J, Saeki N, Sasaki H, Grant SF, Iolascon A, Mosse YP, Cole KA, Li H, Devoto M, McGrady PW, London WB, Capasso M, Rahman N, Hakonarson H, Maris JM: Integrative genomics identifies lmo1 as a neuroblastoma oncogene. Nature 2011;469:216-220.

32 Bartel DP: Micrornas: Genomics, biogenesis, mechanism, and function. Cell 2004;116:281-297. 